\title{
Frecuencia de Neoplasias en Caninos en Quito, Ecuador
}

\author{
Frequency of Neoplasms in Canines in Quito, Ecuador
}

\author{
R.L. Vinueza ${ }^{1,3,4}$, F. Cabrera ${ }^{1}$, L. Donoso ${ }^{1}$, J. Pérez ${ }^{2}$, R. Díaz ${ }^{1}$
}

\section{Resumen}

En Quito, Ecuador, no se dispone de información estadística sobre la prevalencia de neoplasias en caninos, ni su riesgo asociado a edad, sexo y raza. En el presente estudio, a partir de 13573 historias clínicas recabadas entre 2011 y 2014, se identificaron los animales que fueron diagnosticados mediante citología e histopatología como positivos a algún tipo de neoplasia. La prevalencia de tumoraciones en la población fue de 4.94\%, siendo más frecuente en tejidos blandos (39.3\%), piel y anexos (24.4\%), y en hembras, en la glándula mamaria (14.3\%). El riesgo relativo de desarrollar tumores fue cuatro veces mayor en animales de seis o más años de edad; asimismo, los machos son dos veces más predisponentes que las hembras a presentar tumoraciones en piel y anexos. Los animales de razas puras y mayores de cuatro años tendrían hasta $70 \%$ mayor probabilidad de desarrollar tumores que animales mestizos de edad similar.

Palabras clave: perros, tumores, cáncer, Quito

\section{Abstract}

Statistical data of prevalence of neoplasms and risks in canines associated to age, sex and breed are not available in Quito, Ecuador. In the present study, 13573 medical records from 2011 to 2014 were evaluated to identify animals diagnosed by cytology and histopathology as positive for some type of neoplasm. The prevalence of tumors in the population was $4.94 \%$, and the most frequent location was in soft tissues $(39.3 \%)$, skin

${ }^{1}$ Escuela de Medicina Veterinaria, Universidad San Francisco de Quito, Quito, Ecuador

${ }^{2}$ Facultad de Ciencias Químicas y de la Salud, Universidad Técnica de Machala, Machala, Ecuador

${ }^{3}$ E-mail: rvinueza@usfq.edu.ec

${ }^{4}$ Financiamiento principal: Small Grant, Hospital de Especialidades Veterinarias USFQ

Recibido: 14 de abril de 2016

Aceptado para publicación: 23 de noviembre de 2016 
and annexes (24.4\%) and in females, in the mammary gland (14.3\%). The relative risk of developing tumors was four times higher in animals older than 6 years of age; moreover, the predisposition of males to develop tumours skin and annexes was two times higher than in females. Purebred animals older than four years of age are up to $70 \%$ more likely to develop tumors than crossbred dogs of similar age.

Key words: dogs, tumors, cancer, Quito

\section{INTRODUCCIÓN}

La neoplasia se define como «crecimiento nuevo»y corresponde a un proceso patológico caracterizado por una masa de tejido que crece de manera anormal (Trigo y Valero, 2004; Mitchell et al., 2007) por proliferación celular excesiva, indefinida e independiente de los mecanismos de control inhibitorios normales (Cruz, 1997). Este crecimiento celular, clínicamente conocido como tumor o masa, puede tener un comportamiento biológico benigno o maligno (Mitchell et al., 2007).

Las publicaciones existentes sobre prevalencia, tipo de tumor, distribución poblacional y análisis de riesgo de neoplasias en caninos se han realizado principalmente en los Estados Unidos y Europa (McVean et al., 1978; Dobson et al., 2002; Brønden et al., 2007; Merlo et al., 2008), con resultados que difieren de país a país. En América Latina, la información es mayormente provista por clínicas veterinarias (Cruz, 1997; De Nardi et al., 2002; Bravo et al., 2010), algunas veces sin ser específica y con valores que difieren entre sí. Algunos trabajos como los realizados en México y Cuba (Fajardo et al., 2013; Torres et al., 2015) permiten comprender las afecciones de los tumores por raza, edad y sexo de los caninos, así como los factores de riesgo que se relacionan a la ocurrencia de estas patologías.

En la ciudad de Quito existe la percepción del aumento de neoplasias en caninos, sobre todo del tipo cutáneo; sin embargo, no se cuenta con información estadística que permita comprender el fenómeno. El objetivo de este estudio fue establecer la prevalencia de tumoraciones y el riesgo relativo en función de la raza, edad y sexo de los caninos, así como la frecuencia de la ubicación anatómica de los tumores.

\section{Materiales y Métodos}

El estudio fue de tipo epidemiológico descriptivo; sin embargo, los datos permitieron realizar un análisis de Riesgo Relativo bajo el esquema Caso Control de Base Poblacional. En la selección de los sitios de atención veterinaria se tuvo en consideración aquellos que mantenían similares métodos de diagnóstico para tumores que el Hospital Docente de Especialidades Veterinarias de la Universidad San Francisco de Quito, Ecuador, y que además contaban con la participación del patólogo adscrito de la universidad.

La base de información fueron las fichas médicas de pacientes caninos entre junio de 2011 y mayo de 2014, de cinco clínicas veterinarias y del hospital docente indicado. La información fue recolectada $y$ sistematizada al final de cada año académico con ayuda de estudiantes del curso de epidemiología veterinaria. Información repetida e incompleta fue descartada. La muestra poblacional fue de 13573 historias clínicas.

Los casos considerados positivos fueron aquellos diagnosticados a partir de inspección macroscópica, diagnóstico citológico, aspiración o aposición del tumor, mientras que 
Cuadro 1. Distribución porcentual de casos de neoplasias ${ }^{1}$ distribuidos según sexo y raza

\begin{tabular}{lcccccc}
\hline & \multicolumn{2}{c}{ Mestizos } & \multicolumn{2}{c}{ De raza } & \multicolumn{2}{c}{ Total } \\
\hline & $\begin{array}{c}\text { Población } \\
(\mathrm{n})\end{array}$ & $\begin{array}{c}\text { Con } \\
\text { neoplasia } \\
(\%)\end{array}$ & $\begin{array}{c}\text { Población } \\
(\mathrm{n})\end{array}$ & $\begin{array}{c}\text { Con } \\
\text { neoplasia } \\
(\%)\end{array}$ & $\begin{array}{c}\text { Población } \\
(\mathrm{n})\end{array}$ & $\begin{array}{c}\text { Con } \\
\text { neoplasia } \\
(\%)\end{array}$ \\
\hline Machos & 1543 & 4.47 & 6312 & 4.72 & 7855 & 4.6 \\
Hembras & 1126 & 4.26 & 4592 & 5.57 & 5718 & 5.31 \\
\hline Total & 2669 & 4.38 & 10904 & 5.08 & 13573 & 4.94 \\
\hline
\end{tabular}

*Casos confirmados a través de histopatología

el examen histopatológico se efectuó a través de escisión quirúrgica. Las muestras colectadas por el personal de las clínicas fueron fijadas en formol al $10 \%$ y procesadas mediante protocolos rutinarios de inclusión en parafina, corte en secciones de $5 \mu \mathrm{m}$ y tinción con Hematoxilina-Eosina. Todas las muestras fueron examinadas por un solo patólogo.

La asociación entre las variables frecuencia de casos y edad de los animales fue determinada aplicando un modelo lineal a través del programa Statgraphics Centurion v. 16.1. La relación entre las variables raza (pura, mestizo), edad y sexo fue calculada mediante regresión logística. Asimismo, para comparar la frecuencia de las patologías por sistema se aplicó la prueba Chi cuadrado de Pearson de independencia. La medida epidemiológica de Riesgo Relativo (RR) fue aplicada considerando las variables raza, edad y sexo como factor de exposición. El RR es un indicador que permite establecer la fuerza de asociación entre un factor de riesgo y la presencia de la enfermedad comparando la incidencia de los individuos expuestos y los no expuestos (Jaramillo, 2010).

Para el cálculo se utilizó el programa STATA v. 11.1 a partir de los comandos logistic regression y Risk Analysis.

\section{Resultados}

De los 13573 caninos llevados a consulta, 19.7\% (2699) correspondieron a mestizos y el $80.3 \%$ a animales de raza, donde se pudieron identificar hasta 89 razas. Entre estos últimos, $16.7 \%$ fueron Schnauzer, $12.9 \%$ Labrador, 9.6\% French Poodle y 7.1\% Pastor Alemán, mientras que cada una del resto de razas presentaron menos del $4 \%$ de frecuencia, algunas de ellas con apenas 3 a 10 ejemplares. Las neoplasias fueron identificadas en 54 de las 89 razas y en un total de 671 animales entre machos y hembras.

Los 671 casos de neoplasias diagnosticados corresponden a una prevalencia de $4.94 \%$ en la población en estudio. De estos, el $54.7 \%$ correspondió a machos y el $45.3 \%$ a hembras. La frecuencia según el grupo racial y sexo se presenta en el Cuadro 1 . No se encontró diferencia significativa al comparar los valores según grupo racial y sexo (Cuadro 2).

La relación frecuencia de casos y edad fue analizada a través de un modelo lineal, siendo el Coeficiente de Determinación, $\mathrm{R}^{2}$ $=0.96(p<0.001)$, el cual indica un alto nivel de asociación. En el análisis de Regresión 
Cuadro 2. Comparación de Riesgo Relativo ${ }^{1}$ para presentar neoplasias según grupo racial y sexo

\begin{tabular}{lcc}
\hline $\mathrm{H}^{1}$ (Hipótesis alternativa) & Riesgo relativo & IC 98\% \\
\hline $\begin{array}{l}\text { La población de caninos mestizos presenta mayor } \\
\text { frecuencia de tumores que los puros }\end{array}$ & 0.862 & $0.68-1.08$ \\
$\quad \begin{array}{l}\text { Los caninos machos mestizos presentan más } \\
\text { tumores que los de raza pura }\end{array}$ & 0.947 & $0.69-1.28$ \\
$\quad \begin{array}{l}\text { Los caninos hembras mestizos presentan más } \\
\text { tumores que los de raza pura }\end{array}$ & 0.764 & $0.53-1.09$ \\
\hline${ }^{1}$ Incidencia de los expuestos $v$ incidencia en no expuestos & &
\end{tabular}

Logística no se encontró significancia estadística para la interacción de las variables frecuencia de casos, edad y raza; sin embargo, al comparar edad y grupo racial animal puro o mestizo, se obtuvo un resultado significativo en los grupos de cuatro años en adelante $(\mathrm{RR}=0.5$ y 0.7$)$; es decir, un perro de raza pura tiene $50-70 \%$ más probabilidad de desarrollar procesos tumorales a partir de los cuatro años que un perro mestizo de edad similar.

La prevalencia de neoplasias en animales menores de seis años fue del $2.12 \%$, mientras que en mayores de seis años fue 5.14 veces mayor $(10.9 \%)$.

La mayor parte de los tumores se presentó en tejidos blandos (39.3\%), seguido por tumores en piel y anexos (24.4\%) y glándula mamaria (15.1\%), de los cuales cuatro casos fueron detectados en machos (Cuadro 3). Con excepción de los casos reportados en glándula mamaria, sistema cardiovascular e hígado y páncreas, la frecuencia de presentación fue significativamente mayor $(p<0.001)$ en machos que en hembras (Cuadro 4).

La presentación de tumores en tejidos blandos $(57.0 \%$, en hembras y $43.0 \%$ en machos) se registró en 43 de las 89 razas registradas en el estudio. Sin embargo, no se pudo asociar estos tumores con alguna raza
Cuadro 3. Frecuencia de tumores según ubicación anatómica y sexo de caninos en Quito, Ecuador (20112014) $(n=671)$

\begin{tabular}{lcc}
\hline Ubicación anatómica & Macho & Hembra \\
\hline Tejidos blandos & 18.19 & 20.42 \\
Piel y anexos & 6.4 & 18.03 \\
Sistema músculo & 2.1 & 4.62 \\
esquelético & & 2.98 \\
Sistema reproductor & 0.9 & 2.38 \\
Región anal & 0 & 1.64 \\
Sistema linfático & 0.4 & 1.34 \\
Orofaringe & 0.9 & 0.75 \\
Glándula mamaria & 14.3 & 0.3 \\
Hígado, páncreas y & 0.45 & 0.3 \\
ductos & 0.6 & 0.3 \\
Ojos y anexos & 0.75 & 0.3 \\
Sistema respiratorio & 0.1 & 0 \\
Sist. cardiovascular & 0.4 & 1.04 \\
Otros &
\end{tabular}

en especial, debido al reducido número de casos por raza. Por otro lado, los tumores en piel y anexos se presentaron en ejemplares de 30 razas, con una diferencia significativamente mayor para machos con respecto a hembras (Cuadro 4). 
Cuadro 4. Comparación de Riesgo Relativo de presentar neoplasias en piel y anexos y en tejidos blandos en caninos según grupo racial y sexo (Quito, Ec uador)

\begin{tabular}{lcc}
\hline Variable & Riesgo relativo & Valor de $P$ \\
\hline $\begin{array}{l}\text { Riesgo de presentar cualquier tipo de neoplasias por } \\
\text { grupos de edad }(>0<6 \text { años) }\end{array}$ & 5.19 & 0.000 \\
$\begin{array}{l}\text { Riesgo de tumoraciones en tejidos blandos por sexo } \\
\begin{array}{l}\text { Riesgo de tumoraciones en tejidos blandos por grupos } \\
\text { de edad }(>0<6 \text { años) }\end{array}\end{array}$ & 0.78 & 0.045 \\
$\begin{array}{l}\text { Riesgo en piel y anexos por sexo } \\
\text { Riesgo en piel y anexos por grupos de edad }(>0<6\end{array}$ & 2.04 & 0.000 \\
años)
\end{tabular}

\section{Discusión}

Debido a la elevada frecuencia de consultas por neoplasias en caninos, existe la percepción por parte de los profesionales que se están incrementando los casos, tal y como lo mencionan Brønden et al. (2010) y Willard (2012). Este incremento podría explicarse, en parte, por los avances en la atención veterinaria, los cuales contribuyen al aumento en la esperanza de vida de las mascotas (Laflamme, 2012; Niessen et al, 2012), ya que el avance de la edad del animal eleva la probabilidad de desarrollar neoplasias (San Martín, 2005). Fajardo et al. (2013) en México reportaron $59.3 \%$ de tumores en animales entre 7 y 15 años, aunque también encontraron neoplasias en canes de 1 a 2 años. Por otro lado, en el presente estudio se pudo constatar que perros de raza mayores de cuatro años tuvieron $50-70 \%$ mayor probabilidad de presentar neoplasias en relación con perros cruzados de edad similar.

En cuanto a la frecuencia de tumoraciones por sexo, llama la atención que salvo en glándula mamaria, sistema cardiovascular e hígado y páncreas, la frecuencia de casos en machos fue significativamente mayor que en hembras $(p<0.001)$, especialmente en piel y anexos. Esta circunstancia podría explicarse por el hecho de que los animales machos son destinados con mayor frecuencia a labores de guardianía que las hembras y quizás estarían más expuestos a los factores ambientales como los rayos ultravioleta. El Monitor Nacional de Radiación Ultravioleta-EXA (http:/ /uv.exa.ec/infografia.htm) reportó en 2015 que en la ciudad de Quito se registran valores que alcanzan los 24 UVI (índice ultravioleta), valor extremadamente alto según la Organización Mundial de la Salud (OMS, 2003).

En este estudio se obtuvieron valores de frecuencia que difieren en gran medida de los encontrados en estudios realizados en los países vecinos de Colombia y Perú (Ferreira et al., 1997; Vivero et al., 2013). La edad y la raza del animal han sido identificadas como factores de riesgo (Kelsey et al., 1998; Moe et al., 2008), y la afición por adquirir perros de raza pura es cada vez mayor en la ciudad de Quito. Pocos estudios han comparado la ocurrencia de neoplasias entre caninos de raza y mestizos; sin embargo, los estudios revisados (Vail y MacEwen, 2000; Liu y Mikaelian, 2003) indican que la frecuencia de casos debería ser mayor en animales de raza pura que en mestizos; por ejemplo, 
Vascellari et al. (2009) afirman que tanto en perros como en gatos de raza pura se registra el doble de incidencia de tumores malignos, en comparación con animales mestizos. No obstante, si solo se consideran dos grupos raciales (mestizos $v s$ raza pura), el presente estudio indica que no habría diferencia estadística en la ciudad de Quito.

Por otro lado, se han realizado estudios donde se menciona la predisposición de animales mestizos a desarrollar neoplasias específicas (Bravo et al., 2010); por ejemplo, Salamanca et al. (2008) mencionan que el Tumor Venéreo Transmisible (TVT) tiene una mayor incidencia en perras mestizas que en las de raza; sin embargo, estos casos no se explicarían por factores raciales sino por una condición de tenencia, ya que en ciudades como Quito, a los animales mestizos se les permite salir y deambular por la calle, lo que aumenta la probabilidad de contagiarse con el TVT y otras enfermedades. No obstante, los casos encontrados de TVT en este estudio fueron insignificantes, situación que podría ser tema de otro estudio.

E1 $14.3 \%$ de frecuencia de neoplasias en glándula mamaria de hembras difiere de lo señalado por otros estudios, donde se mencionan valores de 20 a 50\% (Zaldívar et al., 2002; San Martín, 2005; Rodríguez et al., 2009). En este estudio, se percibió un factor de protección del $23 \%$ para las hembras mestizas en comparación con hembras de raza pura. Es evidente que mientras las hembras mestizas son habitualmente castradas para evitar que se escapen durante el celo y se reproduzcan, las hembras de raza pura son destinadas a producir cachorros para la venta, incluso después de los seis años de edad. En ese sentido, la ovariohisterectomía probablemente constituiría un factor de protección, al menos, en lo referente a la aparición de tumores mamarios.

Aunque existen diferencias, tanto entre las tipologías de cáncer reportadas como en la metodología de recolección e inclusión de los datos (Brønden et al., 2010), resulta útil comparar los resultados de centros de registro de neoplasias con los hallazgos de esta investigación. Los tumores cutáneos son los primeros por su incidencia en el perro (700 casos/100 000/año) Según el tipo histológico, los tumores cutáneos más frecuentes son los mastocitomas, los adenocarcinomas y los carcinomas de células escamosas (Dorn, 1976). Los denominados tumores de tejidos blandos constituyen un grupo variado de neoplasias provenientes de células del tejido conectivo, que corresponden al tipo C49 de acuerdo al código topográfico internacional (International Classification of Diseases for Oncology [ICD-O-3]) (Egevad et al., 2007).

El hecho de que la ciudad de Quito se encuentre sobre los $2800 \mathrm{msnm}$ y reciba niveles extremos de radiación ultravioleta constituye un factor de riesgo permanente para el desarrollo de ciertas formas de cáncer de piel en animales y personas. Kristen et al. (1992) evaluaron en el hemisferio norte cerca de 1000 perros Beagle expuestos a la radiación ultravioleta, encontrando $17.7 \%$ de individuos con tumores de la piel, principalmente en el abdomen, frecuencia inferior al $24.4 \%$ encontrado en este estudio.

Los centros de atención veterinaria pueden contribuir a la vigilancia epidemiológica pasiva de enfermedades; sin embargo, se requiere estandarizar los métodos de diagnóstico. En el Distrito Metropolitano de Quito existen más de 70 clínicas veterinarias registradas (MDPQ, 2011) y aunque no se cuenta con datos censales, se estima una población de 312500 canes (Andrango y Morales, 2013). Por otro lado, se desconoce el número de animales llevados a consulta veterinaria. Otro elemento relevante es que no se conoce el tipo de diagnóstico utilizado en las clínicas veterinarias, ya que no existe un protocolo unificado.

Conclusiones

- La prevalencia de neoplasias caninas en una población de 13573 casos clínicos 
- entre 2011 y 2014 en la ciudad de Quito, Ecuador, fue de $4.94 \%$, donde el $55.2 \%$ de las neoplasias se presentó en machos y el restante en hembras.

- No se encontraron diferencias significativas sobre la ocurrencia de neoplasias entre canes de razas puras y mestizos; sin embargo, la evaluación del grupo racial con edad indicó que existe una mayor probabilidad (50-70\%) de que perros de razas puras desarrollen tumoraciones a partir de los seis años de edad, en comparación con perros mestizos.

- La mayor frecuencia de neoplasias se registró en tejidos blandos (20.4 y 18.2\% en machos y hembras, respectivamente) y en piel y anexos ( 18.3 y $6.4 \%$ en machos y hembras, respectivamente). Asimismo, la frecuencia de tumores en glándula mamaria en hembras fue del $14.3 \%$ del total de neoplasias.

\section{Literatura Citada}

1. Andrango ML, Morales GV. 2013. Identificación de las especies de pulgas y endoparasitosis gastrointestinales asociadas en caninos de tres parroquias de la zona urbana (El Condado, San Juan y Quitumbe) del D.M.Q. Tesis de Médico Veterinario Zootecnista. Quito: Univ Central del Ecuador. $130 \mathrm{p}$.

2. Bravo D, Cruz-Casallas P, Ochoa J. 2010. Prevalence of neoplasm in canines in the University of the Llanos, during 2004 to 2007. Rev MVZ Córdoba 15: 1925-1937.

3. Bronden LB, Flagstad A, Kristensen AT. 2007. Veterinary cancer registries in companion animal cancer: a review. Vet Comp Oncol 5: 133-144. doi: 10.1111/ j.1476-5829.2007.00126.x

4. Bronden LB, Nielsen SS, Toft N, Kristensen AT. 2010. Data from the Danish Veterinary Cancer Registry on the occurrence and distribution of neoplasms in dogs in Denmark. Vet Rec 166: 586-590. doi: 10.1136/vr.b4808
5. Cruz R. 1997. Clasificación histológica según la organización mundial de la salud (OMS) y frecuencia de neoplasias en animales domésticos, aves y peces, durante el periodo 1967-1995 en Valdivia, Chile. Tesis de Grado. Valdivia, Chile: Univ Austral de Chile. 134 p.

6. De Nardi AB, Rodaski S, Sousa RS, Costa TA, Macedo TR, Rodigheri SM, Rios A, Piekarz CH. 2002. Prevalência de neoplasias e modalidades de tratamentos em cães, atendidos no Hospital Veterinário da Universidade Federal do Paraná. Arch Vet Sci 7: 15-26. doi: 10.5380/avs.v7i2.3977

7. Dobson JM, Samuel S, Milstein H, Rogers K, Wood JLN. 2002. Canine neoplasia in the UK: estimates of incidence rates from a population of insured dogs. J Small Anim Pract 43: 240-246.

8. Dorn CR. 1976. Epidemiology of canine and feline tumors. J Am Anim Hosp Ass 12: 307-312.

9. Egevad L, Heanue M, Berney D, Fleming $K$, Ferlay $K .2007$. Histological groups. In: Curado MP, Edwards B, Shin HR, et al. (eds). Cancer incidence in five continents. Vol IX. Scientific Publication No. 160. Lyon, France: International Agency for Research on Cancer. p 101-121.

10. Fajardo R, Alpízar A, Pérez LS, Martínez JS, Córdova E. 2013. Prevalence of tumors in dogs from the municipality of Toluca, México, from 2002 to 2008. Arch Med Vet 45: 305-309.

11. Ferreira G, Pedraza O, Arango M. 1997. Neoplasias de glándula mamaria canina diagnosticadas en Medellín, Colombia, entre 1968 y 1994. Vet Mex 28: 257-259.

12. Jaramillo CJ. 2010. Epidemiología veterinaria. México: El Manual Moderno. $198 \mathrm{p}$.

13. Kelsey JL, Moore AS, Glickman LT. 1998. Epidemiologic studies of risk factors for cancer in pet dogs. Epidemiol Rev 20: 204-217. 
14. Kristen J, Nikula SA, Benjamin GM, Angleton W, Saunders J, Lee AC. 1992. Ultraviolet radiation, solar dermatosis, and cutaneous neoplasia in beagle dogs. Radiat Res 129: 11-18.

15. Laflamme DP. 2012. Nutritional care for aging cats and dogs. Vet Clin North Am Small Anim Pract 42: 769-791. doi: 10.1016/j.cvsm.2012.04.002

16. Liu SM, Mikaelian I. 2003. Cutaneous smooth muscle tumors in the dog and cat. Vet Pathol 40: 685-692. doi: 10.1354/ vp.40-6-685

17. McVean DW, Monlux AW, Anderson PS, Silverg SL, Roszel JF. 1978. Frequency of canine and feline tumors in a defined population. Vet Pathol 15: 700-715.

18. [MDPQ] Dirección de Planificación del Municipio del Distrito Metropolitano de Quito. Quito al Día. 2011. Ordenanza Metropolitana No. 048. Tenencia, Protección y Control de Fauna Urbana del 15 de abril de 2011. [Internet]. Disponible en: http://www.quito.gob.ec/ index.php/servicios/sitios-seguros/55otros-servicios/152-ordenanza-municipal-n-048

19. Merlo DF, Rossi L, Pellegrino $C$, Ceppi M, Cardellino U, Capurro C, Ratto A, et al. 2008. Cancer incidence in pet dogs: findings of the Animal Tumor Registry of Genoa, Italy. J Vet Intern Med 22: 976-984. doi: 10.1111/j.19391676.2008.0133.x

20. Mitchell R, Kumar V, Abbas A, Fausto N. 2007. Patología estructural y funcional - Robbins Cotran. $7^{\mathrm{a}}$ ed. España: Elsevier. $1464 \mathrm{p}$.

21. Moe L, Gamlem H, Dahl K, Glattre E. 2008. Canine neoplasia - populationbased incidence of vascular tumours. APMIS Supl 116(125): 63-68.

22. Niessen SJ, Powney S, Guitian J, Niessen AP, Pion PD, Shaw JA, Church DB. 2012. Evaluation of a quality-of-life tool for dogs with diabetes mellitus. J Vet Intern Med 26: 953 -
961. doi: 10.1111/j.1939-1676.2012. 00947.x

23. [OMS] Organización Mundial de la Salud. 2003. Radiación ultravioleta y el Programa INTERSUN. Índice UV Solar Mundial. [Internet]. Disponible en: http://www.who.int/uv/publications/ globalindex/es/

24. Rodríguez BJ, Ortiz LC, Garzón A, Gómez LF, Vásquez L. 2009. Valoración de la citología para el diagnóstico de tumores en caninos. Rev Colomb Cienc Pecu 22: 42-53.

25. Salamanca $S$, Santander-Baquero A, Triana-García $P A$, Romero $S$, Rondón-Barragán IS. 2008. Tumor venéreo transmisible (TVT) con metástasis pulmonar: reporte de caso. Orinoquia 12: 162-170.

26. San Martín MA. 2005. Neoplasias caninas, evaluación estadística 1990-1994. Tesis de Médico Veterinario. Lima: Univ Nacional Mayor de San Marcos. 50 p.

27. Trigo F, Valero G 2004. Patología general veterinaria. $4^{\mathrm{a}}$ ed. México: McGraw Interamericana. $468 \mathrm{p}$.

28. Torres M, Peraza B, Fabré $Y$, Rodríguez J, Calaña L, Márquez M, Zamora Y, et al. 2015. Frecuencia de presentación de neoplasias en caninos del municipio San Miguel del Padrón, La Habana, Cuba. Rev Salud Anim 37: 39-46.

29. Vail DM, MacEwen EG. 2000. Spontaneously occurring tumors of companion animals as models for human cancer. Cancer Invest 18: 781-792. doi: 10.3109/07357900009012210

30. Vascellari M, Baioni E, Ru G, Carminato A, Mutinelli F. 2009. Animal tumour registry of two provinces in northern Italy: incidence of spontaneous tumours in dogs and cats. BMC Vet Res 5: 39-47. doi: 10.1186/1746-6148-5-39

31. Vivero LE, Chavera A, Perales $R$, Fernández V. 2013. Frecuencia de neoplasias caninas en Lima: estudio retrospectivo en el periodo 1995-2006. Rev Inv Vet Perú 24: 182-188. doi: 10.15381/ rivep.v24i2.2487 
32. Willard MD. 2012. Alimentary neoplasia in geriatric dogs and cats. Vet Clin North Am Small Anim Pract 42: 693-706. doi: 10.1016/j.cvsm.2012.04.006

33. Zaldívar N, Puebla H, Ferrales $Y$, Almaguer Y, Vaillant Y. 2002. Impor- tancia de las neoplasias en los animales domésticos. Neoplasias diagnosticadas en los centros de epizootiología y diagnóstico veterinario de Guantánamo y Granma, Cuba. Rev Prod Anim 14: 51:53. 